\title{
Postoperative sellar hematoma after pituitary surgery: clinical and biochemical characteristics
}

\author{
Nadine El-Asmar', Katia El-Sibai', Ribal Al-Aridi', Warren R Selman² and \\ Baha M Arafah' \\ 'Division of Clinical and Molecular Endocrinology and ${ }^{2}$ The Department of Neurological Surgery, \\ Case Medical Center and Case Western Reserve University, Cleveland, OH, USA
}

Correspondence
should be addressed
to Baha M Arafah
Email
baha.arafah@case.edu

\begin{abstract}
Background: Postoperative sellar hematoma can develop following surgery for pituitary tumors and other sellar masses such a Rathke's cleft cyst (RCC) due to continued blood oozing. Though often mild sellar hematoma can create mass effects that might impair pituitary function.

Aim: This study summarizes the clinical and biochemical characteristics of sellar hematoma and explores the potential mechanisms for the associated hypopituitarism.

Patients and methods: Sellar hematoma was suspected clinically (worsening headaches, visual impairment, and mental status alterations) and confirmed radiographically in 23 patients (18/279 with macroadenomas and in 5/92 with RCC).

Postoperatively, patients were monitored without glucocorticoid therapy and all had appropriately normal HPA function before sellar hematoma diagnosis.

Results: The demographics of patients who had sellar hematoma were similar to those who did not. Biochemical changes at diagnosis included decreased plasma ACTH and its dependent steroids (cortisol, DHEA, and DHEA-S), concomitant mild hyperprolactinemia, and mild hyponatremia $(P<0.005$ for all parameters). Treatment with glucocorticoids resulted in rapid clinical improvement in most patients. Glucocorticoid therapy was discontinued within 2 weeks and re-testing thereafter showed normal HPA function in 16/23. None of the patients without sellar hematoma had worsening in pituitary function, visual, or neurological symptoms.

Conclusions: When large, sellar hematoma can lead to mass effect that causes headaches, visual symptoms, and acute and often reversible hypopituitarism manifesting initially as impaired HPA function. Rapid resolution is observed in most patients with glucocorticoid administration The rapid onset and reversibility of hypopituitarism associated with mild hyperprolactinemia suggest that compression of pituitary stalk/ portal vessels is likely the dominant mechanism of pituitary dysfunction in this setting.
\end{abstract}

\section{Introduction}

Transsphenoidal pituitary adenomectomy is the treatment of choice for most pituitary tumors, especially those that are nonfunctional. In experienced hands, normal pituitary function is most often preserved (1) and recovery of lost function is common $(2,3)$. Recovery of pituitary function can be documented to occur immediately after
() 2016 European Society of Endocrinology Printed in Great Britain adenomectomy (3) even in patients with pituitary tumor apoplexy $(4,5)$.

A similar approach is used for the resection of other sellar masses such as Rathke's cleft cyst (RCC) which is a non-neoplastic cystic mass that develops early in life as a result of poor obliteration of the Rathke's pouch $(6,7,8,9)$. 
Although most RCCs remain small and asymptomatic throughout life, some become large, resulting in a mass effect on the pituitary and surrounding structures that are difficult to distinguish from a pituitary macroadenoma $(6,7,8,9)$. When large, the clinical manifestations of RCC include headaches, visual symptoms, and variable degrees of hypopituitarism $(6,7,8,9)$. Treatment of this condition becomes necessary in symptomatic patients and is primarily surgical. Since most RCCs are intrasellar, transsphenoidal surgery is often the treatment of choice for a large and symptomatic RCC.

As stated earlier, transsphenoidal decompressive surgery for pituitary tumors or RCC is generally well tolerated with minimal morbidity and mortality. The complication rate is usually low in experienced hands, and is influenced by size and location of the adenoma, as well as the patient's medical condition $(10,11,12)$. Commonly encountered side-effects or complications include diabetes insipidus in $5-15 \%$, which is often transient, and less commonly infection and CSF leak $(10,11,12)$. The development of hypopituitarism following transsphenoidal pituitary surgery is rare when the surgical procedure is performed by experienced surgeons. The time of onset of hypopituitarism in the latter setting is often within a few hours after the procedure and is attributed to direct injury and/or ischemic event during surgery. If this were the case, one would not expect recovery of that function at any time in the future.

Although surgeons always endeavor to ensure adequate homoeostasis at the end of surgical resection of adenomas or RCC, variable degrees of hemorrhage may recur at the surgical site in some patients. Subsequently, postoperative hematoma can develop following transsphenoidal pituitary surgery. Although it is often mild and thus, clinically unrecognized, it can at times be large enough to create a mass effect on the normal pituitary and, therefore, interfere with pituitary function. In addition to the development of hypopituitarism, sellar hematomas can potentially lead to a mass effect that can compress surrounding perisellar structures which can potentially lead to the development of headaches, visual disturbances, and cranial nerve palsies. Postoperative sellar hematoma is not a well-recognized entity and to date, there have been only a few case reports $(13,14,15)$. We are not aware of any published reports of a sellar hematoma developing after transsphenoidal surgical decompression of an RCC. Specifically, there are no reports on the prevalence of this complication and its impact on pituitary function.

The current investigation provides a detailed review of the clinical and biochemical characteristics in patients who were demonstrated to have a postoperative sellar hematoma and a comparison to the features observed in those who did not develop this complication. We postulated that the development of postoperative sellar hematoma results in a mass effect that can lead to the compression of perisellar structures including the portal vessels and the infundibulum and therefore will be associated with acute loss of adrenal function and mild-to-moderate hyperprolactinemia.

\section{Patients and methods}

To achieve our objectives, we performed a retrospective review of the clinical and biochemical data on all patients with pituitary tumors/masses who had elective transsphenoidal surgery over a 10 -year period (January 2005 to December 2014) and identified those who were clinically suspected and confirmed by imaging studies to have the diagnosis of postoperative sellar hematomas. Patients who presented with the apoplexy syndrome were excluded. As a control group, we reviewed similar data on patients who underwent the same surgical procedure during the same time frame but who did not have symptoms to raise clinical concerns for postoperative hematoma. Pre and perioperative clinical and biochemical data were collected and analyzed in both groups. The primary objectives of this study include determining the prevalence as well as the clinical and biochemical manifestations of clinically significant sellar hematomas after transsphenoidal surgery. A secondary objective of the study was to investigate the potential for recovery of pituitary function in these patients.

As stated earlier, the development of postoperative sellar hematomas after transsphenoidal surgery is not well appreciated, particularly when one looks at the impact on pituitary function. It would be therefore hard to examine this complication prospectively and therefore the current investigation is a retrospective review of patients' data. Fortunately, however, over the past 25 years we have followed an established protocol detailing perioperative management of all patients who underwent surgical resection of pituitary masses. This has allowed us to obtain consistent data at specific perioperative time points in patients who had the sellar hematoma and others who did not. In all cases, transsphenoidal decompression surgery on all patients was performed by the same neurosurgeon (WRS). The surgical procedure in all cases was done via a direct transnasal approach to the sphenoid sinus as described by Griffith and Veerapen (16) with the aid of the operating microscope, and standard means of packing 
with adipose tissue in the sphenoid was performed. Patients with prolactin or ACTH-secreting adenomas were included in assessing the prevalence of this complication but their perioperative prolactin and HPA function data were excluded when the alterations in their respective secretion were examined. As will be discussed in a subsequent section of this manuscript, patients were suspected to have a sellar hematoma primarily on clinical grounds, and the diagnosis was confirmed or excluded on imaging studies. The study was approved by the Institutional Research Board.

\section{Laboratory assays}

Plasma ACTH concentrations were measured using solid-phase two-site sequential chemiluminescent immunometric assay (Immulite) supplied by Sieman Healthcare Diagnostic Products Ltd, Llanberis, Gwynedd U55 4EL, UK. The lower limit of detectability in the assay was $5 \mathrm{ng} / \mathrm{L}$, and the intra- and inter-assay coefficients of variation determined at different ranges in the assays varied from 6 to $10 \%$. The assay utilized two specific antibodies that detect the entire ACTH molecule. Testing by the manufacturer indicated that there was no cross reactivity in this assay system with the ACTH fragment molecules or with ACTH molecule precursors such as $\alpha \mathrm{MSH}$ and pro-opiomelanocortin. The normal AM plasma ACTH in this assay was $8-46 \mathrm{ng} / \mathrm{L}$. Serum DHEA, DHEA-S, and prolactin were measured using established procedures as described previously $(3,17,18)$. Serum total cortisol levels were measured by a chemiluminescence assay technique (Centaur/Bayer Diagnostics, Tarrytown, NY, USA). The lower limit of detectability was $0.2 \mu \mathrm{g} / \mathrm{dL}$, and the inter- and intra-assay coefficients of variation were $7.7 \%$ and $4.3 \%$ respectively $(17,18)$.

\section{Statistical analysis}

Data are presented as mean \pm standard deviation (S.D.), unless otherwise stated. The perioperative data on patients with perisellar hematomas and those without clinical or biochemical evidence for that complication were first analyzed using the Kruskal-Wallis test, as a nonparametric alternative to the analysis of variance test and then comparisons between groups were done using the Wilcoxon rank-sum test for nonparametric measurements. Categorical data were compared using Chi square and Fisher's exact tests. Differences were considered significant when the two-sided $P$ values were less than 0.05. All data analysis was made using SPSS program.

\section{Standard perioperative management protocol}

\section{Clinical monitoring}

Following transsphenoidal surgery, patients are monitored carefully by the Pituitary Service, the neurosurgical team along with highly specialized experienced nurses either in the Neurological ICU or a step-down unit. Monitoring parameters in these patients include, but are not limited to, cardiovascular/respiratory evaluation along with assessments of headache intensity, need for pain medications, mental status, neurological and/or visual symptoms, fluid balance, and urine flow rate. Various degrees of headaches are expected in the majority of patients, and most require pain control during the first 24 hours. Suspicion for postoperative sellar hematoma was raised when one or more of the following clinical symptoms/signs were detected. These include newer or worsening visual complaints (decreased acuity, diplopia; visual field deficits), unexplained alteration in mental status, and persistent severe unrelenting headaches after the first postoperative day despite removal of the nasal packs. Such patients were suspected clinically to have postoperative sellar hematomas. Additional symptoms were observed in these patients, such as fatigue, but that was never the sole manifestation in any patient.

Imaging studies are not routinely done in the immediate postoperative period, but are usually obtained 10-12 weeks after surgery. However, imaging studies were obtained on patients who were suspected to have a sellar hematoma as described herein. In patient with any of the above-mentioned clinical manifestations, a postoperative sellar hematoma was diagnosed when magnetic resonance imaging (MRI) was performed and showed evidence for a sellar mass that has the characteristics of blood/blood products and that resulted in compression of the infundibulum and/or other perisellar structures. An illustrative example of a patient who developed this complication is shown in Fig. 1.

\section{Biochemical monitoring}

Biochemical monitoring is carried out in parallel with that of clinical assessment described above. The details of our standard protocol have been published previously $(17,18)$. The protocol stipulates that glucocorticoids are not administered before and during surgery to patients who had normal hypothalamic pituitary adrenal (HPA) function as recently defined $(17,18)$. Instead, patients were frequently evaluated clinically and biochemically for the evidence of adrenal insufficiency $(3,17,18)$. Blood 

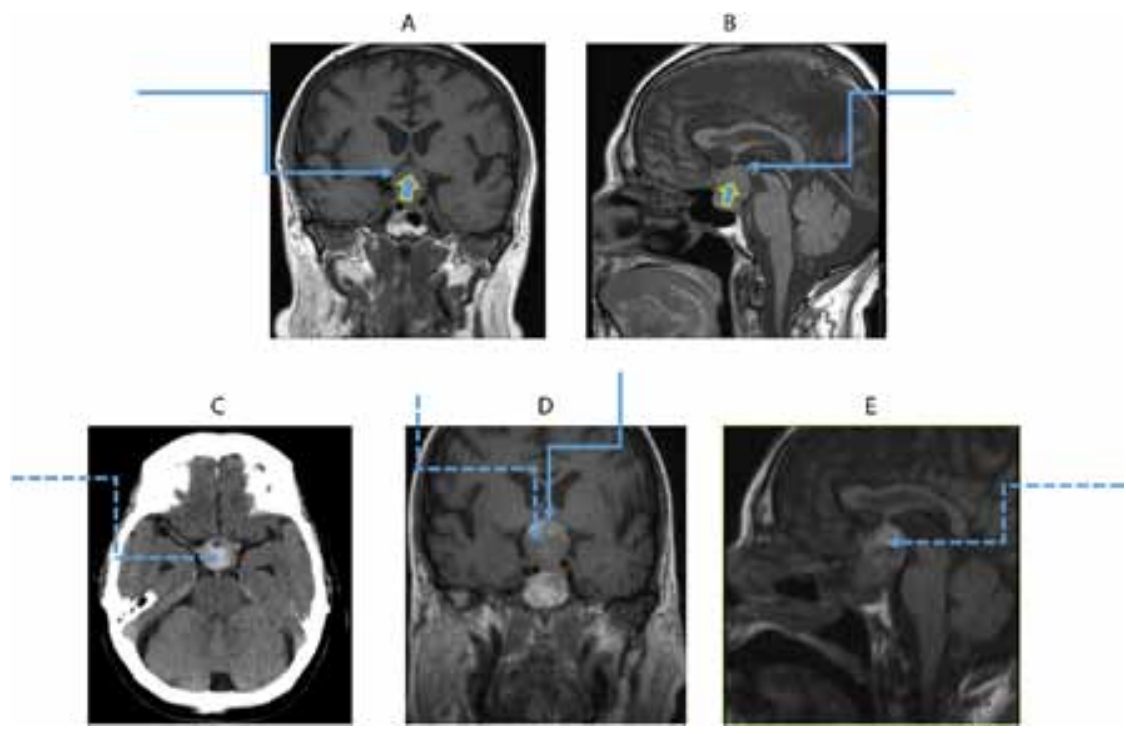

\section{Figure 1}

An illustrative example of a postoperative hematoma in a 73-year-old woman with a pituitary macroadenoma. The preoperative coronal (A) and sagittal (B) noncontrasted views of the tumor (short arrows) are shown. Also shown is the optic chiasm (long uninterrupted arrow). In this patient, postoperative hematoma was suspected $30 \mathrm{~h}$ after surgery and documented by a noncontrasted CT (C) and a noncontrasted coronal (D) and sagittal (E) T-1 MRI performed at 32 and $40 \mathrm{~h}$,

samples for the determination of plasma ACTH, serum cortisol, and prolactin levels were drawn before surgery and at $4,8,12,18,24,36$, and $48 \mathrm{~h}$ after adenomectomy. Serum cortisol levels usually reach a peak $8-10 \mathrm{~h}$ after surgery and return to baseline gradually thereafter. In patients with normal postoperative pituitary adrenal function, the serum cortisol is $>15 \mu \mathrm{g} / \mathrm{dL}$ on multiple occasions during the first 36 postoperative hours. Patients with documented preoperative hypothyroidism are treated with thyroxine preoperatively. However, those with ACTH insufficiency are treated preoperatively with physiological doses of hydrocortisone, and an oral dose of $30 \mathrm{mg}$ was given $2-3$ hours before surgery. No additional glucocorticoids are administered unless the patient exhibits symptoms suggestive of adrenal insufficiency. Thereafter, their serum cortisol and ACTH levels are monitored as stated above without additional glucocorticoids. Serum cortisol levels are usually available within 2-3 hours, which would allow the treating physician to make a decision as to whether glucocorticoids should be administered. The protocol stipulates that glucocorticoids should be administered when the serum cortisol is $<10 \mu \mathrm{g} / \mathrm{dL}$ on more than respectively. The $\mathrm{CT}$ image (C) showed hyperintense suprasellar lesion (Interrupted arrow) that is consistent with acute hemorrhage. Noncontrasted MRI coronal (D) and sagittal (E) images done a few hours after the CT showed areas of hyper and hypo intensities within the sella turcica consistent with recent bleeding (interrupted lines in panels $D$ and E). Compression of the optic chiasm (uninterrupted arrow) by the hematoma is shown in panel $D$.

one occasion during the first 24 postoperative hours. Blood samples are drawn as scheduled until exogenous glucocorticoids are administered. In addition to the above routine measurements, we examined changes in patients' electrolytes at least twice daily for the first 2 postoperative days.

\section{Results}

\section{Prevalence, patients characteristics, and clinical manifestations}

Over a period of 10 years, a total of 399 patients with pituitary masses had transsphenoidal pituitary surgery performed by one neurosurgeon (WRS). Of these, 307 patients had pituitary tumors while 92 had Rathke's cleft cyst (RCC). Postoperative sellar hematoma was diagnosed in 23/399 (5.8\%) of the patients who had transsphenoidal pituitary surgery (Table 1). Of the 307 patients who had pituitary adenomas, 28 had microadenomas $(<1.0 \mathrm{~cm})$ and none of these had postoperative sellar hematoma. In contrast, 18/279 who had macroadenomas $(>1.0 \mathrm{~cm})$ and 5/92 of those with RCC (all were $>1.0 \mathrm{~cm}$ ) had clinically significant postoperative hematomas (Table 1). The records of all 
Table 1 Clinical characteristics of the study groups. The study groups included patients who had and those who did not have sellar hematomas after transsphenoidal pituitary surgery. None of the 28 patients with pituitary microadenomas had a postoperative hematoma.

\begin{tabular}{|c|c|c|c|c|c|c|}
\hline & \multicolumn{3}{|c|}{ Macroadenomas $(n=279)$} & \multicolumn{3}{|c|}{ Rathke's cyst $(n=92)$} \\
\hline & $\begin{array}{c}\text { Postop } \\
\text { hematoma }\end{array}$ & $\begin{array}{l}\text { No postop } \\
\text { hematoma }\end{array}$ & $P^{*}$ & $\begin{array}{c}\text { Postop } \\
\text { hematoma }\end{array}$ & $\begin{array}{l}\text { No postop } \\
\text { hematoma }\end{array}$ & $P^{*}$ \\
\hline Number & 18 & 261 & & 5 & 87 & \\
\hline Tumor Size & $2.7 \pm 1.0$ & $2.5 \pm 1.1$ & NS & $2.6 \pm 0.9$ & $2.4 \pm 1.0$ & NS \\
\hline Tumor Type & & & & N/A & N/A & N/A \\
\hline Gonadotroph & $6 / 18$ & $34 / 279$ & NS & & & \\
\hline Non-secreting & $5 / 18$ & $22 / 279$ & NS & & & \\
\hline $\mathrm{GH}$ & $5 / 18$ & $27 / 279$ & NS & & & \\
\hline Prolactin & $1 / 18$ & $13 / 279$ & NS & & & \\
\hline Silent ACTH & $3 / 18$ & $3 / 279$ & NS & & & \\
\hline TSH & $0 / 18$ & $1 / 279$ & NS & & & \\
\hline \multicolumn{7}{|l|}{ Preoperative impaired pituitary function } \\
\hline Adrenal & $4 / 18$ & $32 / 261$ & NS & $0 / 5$ & $8 / 87$ & NS \\
\hline Thyroid & $4 / 18$ & $35 / 216$ & NS & $2 / 5$ & $11 / 87$ & NS \\
\hline Gonadal & $15 / 18$ & $172 / 261$ & NS & $3 / 5$ & $37 / 87$ & NS \\
\hline Immediate Recovery of HPA & $4 / 4$ & $22 / 32$ & & $0 / 0$ & $5 / 8$ & NS \\
\hline Preoperative use of aspirin/NSAID** & $5 / 18$ & $16 / 261$ & $<0.001$ & $1 / 5$ & $5 / 87$ & $<0.001$ \\
\hline \multicolumn{7}{|l|}{ Symptoms/signs } \\
\hline Severe headaches at $36-48 \mathrm{~h}$ postop & $16 / 18$ & $10 / 261$ & $<0.001$ & $4 / 5$ & $3 / 87$ & $<0.001$ \\
\hline New/worsening visual symptoms & $8 / 18$ & $2 / 261$ & $<0.001$ & $2 / 5$ & $0 / 87$ & $<0.001$ \\
\hline Altered mental status & $5 / 18$ & $4 / 261$ & $<0.001$ & $2 / 5$ & $1 / 87$ & $<0.001$ \\
\hline Photophobia & $3 / 18$ & $3 / 261$ & $<0.001$ & $2 / 5$ & $2 / 87$ & $<0.001$ \\
\hline
\end{tabular}

399 patients were reviewed for documentation of postoperative clinical course and biochemical and imaging studies. As given in Table 1, the prevalence of postoperative hematoma was similar in patients who had macroadenomas $(6.4 \%)$ and those who had large $(>1 \mathrm{~cm})$ RCC $(5.4 \%)$.

The diagnosis of postoperative sellar hematoma was confirmed in all 23 patients 1.5-8 days after surgery (mean: $2.8 \pm 1.1$; median: 2 days). An illustrative example of the imaging features is shown in Fig. 1. The diagnosis was made before the patients were discharged home in $20 / 23$ patients and a few days after discharge in the remain- ing three patients. There were no differences in age, gender, prevalence of hypertension or diabetes nor were there differences in tumor sizes or histopathology between the 23 patients who had sellar hematomas and those who did not (Table 1). Importantly, however, we noted that 6 of the $23(23 \%)$ who had hematomas were taking aspirin or other nonsteroidal agents preoperatively, although these were discontinued more than one week before surgery. In contrast, only $21 / 348(6 \% ; P \leq 0.001)$ patients with similarly large tumors (adenomas and RCC) who did not have clinically significant hematomas were using such medications.

Table 2 Biochemical data obtained 12 and $24 \mathrm{~h}$ after transsphenoidal surgery in patients with pituitary macroadenomas and others with Rathke cleft cyst. The data of patients who had sellar hematoma are compared with those who did not have that complication. The concentration of ACTH, cortisol, DHEA, and DHEA-S obtained at the 12th and 24th postoperative hours in patients who subsequently developed postoperative sellar hematoma was similar to those in subjects who did not develop that complication. In both groups, all levels were appropriately elevated and indicated normal HPA function.

\begin{tabular}{lccc}
\hline & \multicolumn{2}{c}{$\mathbf{1 2 ~ h}$} \\
ACTH $(\mathrm{ng} / \mathrm{L})$ & Hematoma & No hematoma \\
Cortisol $(\mu \mathrm{g} / \mathrm{dL})$ & & $56.2 \pm 23.2$ & $69.3 \pm 23.4$ \\
DHEA $(\mathrm{ng} / \mathrm{ml})$ & $54.1 \pm 8.1$ & & $38.2 \pm 11.1$ \\
DHEA-S $(\mu \mathrm{g} / \mathrm{dL})$ & $131 \pm 79$ & & $6.7 \pm 4.6$ \\
\hline
\end{tabular}

\begin{tabular}{|c|c|}
\hline \multicolumn{2}{|c|}{$24 \mathrm{~h}$} \\
\hline Hematoma & No hematoma \\
\hline $46.7 \pm 14.2$ & $55.1 \pm 22.4$ \\
\hline $21.9 \pm 8.1$ & $24.6 \pm 11.1$ \\
\hline $3.1 \pm 2.3$ & $3.8 \pm 3.4$ \\
\hline $111 \pm 88$ & $129 \pm 98$ \\
\hline
\end{tabular}


The 23 patients had clinical symptoms, physical examination features, and laboratory findings that justified an imaging study of the sella which confirmed the presence of surgical site hematoma. Clinical symptoms that raised concern for the diagnosis of sellar hematoma (Table 1) included worsening headache (21/23), decreased visual acuity (8/23), cranial nerve palsies (7/23), alteration in mental status (7/23), photophobia $(5 / 23)$, fatigue, and nausea (18/23). All 23 patients had more than one of these symptoms/signs, but worsening and/or severe headache was a dominant complaint. Decreased visual acuity and/or cranial nerve palsies were observed in nearly $40 \%$ of the patients. Patients without postoperative hematomas had minimal symptoms at 36-48h (Table 1) and none had new impairment in pituitary function.

\section{Pre and peri-operative pituitary function}

Preoperative pituitary function in the 23 patients who had postoperative hematomas was similar to that in the 348 patients who had large tumors (261 with macroadenomas and 87 with RCC) and did not develop that complication. Specifically, among the subjects who developed sellar hematoma, 6/23 had central hypothyroidism and were treated with thyroxine before and during the immediate postoperative period. Similarly, 4/23 had impaired HPA function preoperatively and were treated with hydrocortisone till the morning of surgery as stipulated in our protocol. All four patients with preoperative ACTH deficiency recovered HPA function within a few hours and were documented to have appropriately elevated ACTH (>100 ng/L) and

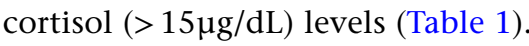

In assessing the alterations in HPA function in patients who developed clinically significant hematoma, we included data on patients $(n=19)$ who had normal function preoperatively and the 4 who recovered function. The data and conclusions would be exactly the same when we exclude the 4 subjects who recovered HPA function. Thus, before the development of hematoma, all 23 patients had clear evidence for normal HPA function. Their plasma ACTH and serum levels of cortisol, DHEA, and DHEA-S measured at 12,24 , and 36 hours after surgery were appropriately elevated and similar to those who did not have a hematoma (Table 2). Furthermore none of these patients had any evidence indicating impairment in ADH secretion as evidenced by the absence of polyuria, neutral fluid balance and a normal serum osmolality, and sodium concentration.

\section{Biochemical features at diagnosis}

Since the diagnosis of sellar hematoma was made over a broad time frame (1.5-8 days after surgery), we assigned day- 0 as the time of diagnosis and examined the data on HPA function the preceding 24-48h (whenever available) to assess changes related to the development of hematoma. In 3 the patients who were diagnosed a few days after discharge, we did not have data on their HPA function at 1 and 2 days. Biochemical features observed at the time of hematoma diagnosis included a significant $(P<0.005)$ decline in plasma ACTH, serum cortisol, DHEA, and DHEA-S as well as a relative increase in serum prolactin levels (Fig. 2) as compared with the respective data obtained 24-48h earlier. Similarly, the mean serum $\mathrm{Na}+$ concentration decreased (from $139.9 \pm 2.9$ at $24 \mathrm{~h}$ before diagnosis to $134 \pm 7.1 \mathrm{meq} / \mathrm{L}$ at diagnosis), while frank hyponatremia ( $\mathrm{Na}$ of $<135 \mathrm{meq} / \mathrm{L})$ was demonstrated in $8 / 23$ patients. At the time of diagnosis of the hematoma, serum cortisol levels were quite low $(<5 \mu \mathrm{g} / \mathrm{dL})$ in $15 / 23$ patients and the remaining 8 patients had levels ranging from 6 to $12.9 \mu \mathrm{g} / \mathrm{dL}$, and all were associated with low or low-normal plasma ACTH levels ranging from $<5$ to $24 \mathrm{ng} / \mathrm{L}$ (Fig. 2).
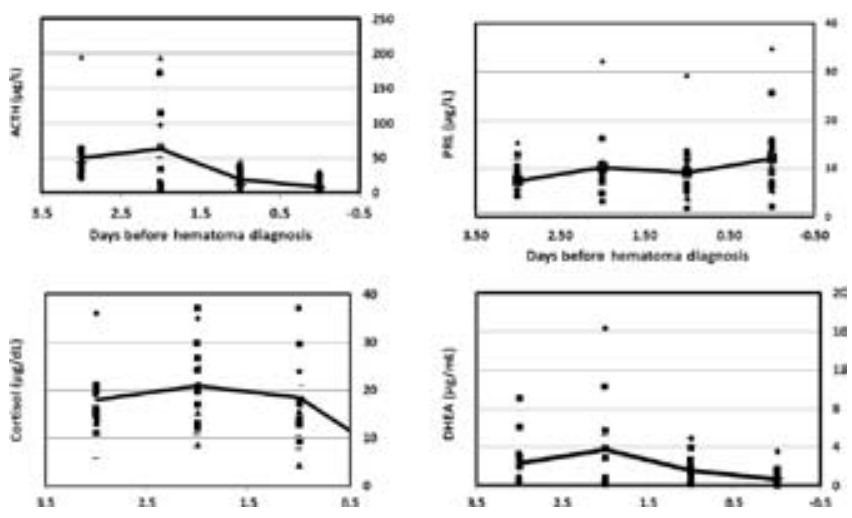

\section{Figure 2}

Serial measurements of ACTH, cortisol, DHEA, and prolactin levels in patients who were demonstrated to have postoperative sellar hematoma. As the onset of symptoms varied, we designated time: 0 as the time when the diagnosis was made and retrospectively looked at the data in the preceding days. In all figures, each marker represents data on a single patient. The line in each graph represents the mean of the data. There was a rapid decline in plasma ACTH followed by a decrease in serum cortisol and DHEA and a rise in serum prolactin levels $(P<0.001)$ at the time of diagnosis when compared with the data obtained 24 hours earlier. 


\section{Management and outcome}

Rapid improvement in symptoms was observed in the majority of patients within $24 \mathrm{~h}$ of the administration of glucocorticoids (hydrocortisone $25 \mathrm{mg}$ every $6 \mathrm{~h}(n=12)$ or dexamethasone $6-12 \mathrm{mg} /$ day given in two or three divided doses $(n=11))$. However, the persistence of symptoms despite $48 \mathrm{~h}$ of glucocorticoid therapy prompted surgical re-exploration in $4 / 23$ patients, in whom the diagnosis was further confirmed visually and histologically. All 23 patients were discharged home 5-10 days after surgery on glucocorticoid therapy that was tapered gradually over a 2-week period. Glucocorticoid therapy was discontinued in these patients after 2-3 days and HPA function was re-tested using morning serum cortisol concentration of $>12 \mu \mathrm{g} / \mathrm{dL}$ indicating normal function and a value of $<5 \mu \mathrm{g} / \mathrm{dL}$ indicating impaired HPA axis $(17,18)$. A lowdose $(1 \mu \mathrm{g})$ cosyntropin test was performed in subjects whose morning serum cortisol level was between 5 and $12 \mu \mathrm{g} / \mathrm{dL}(17,18)$. Following glucocorticoid withdrawal, HPA function was documented to be normal in $17 / 23$ patients and impaired in the remaining 6 patients. The baseline serum cortisol in the 17 patients with normal HPA function ranged from 9.3 to $17 \mu \mathrm{g} / \mathrm{dL}$ (Fig. 3) and the lowest response to $1 \mu \mathrm{g}$ cosyntropin test in those who had it was $23.8 \mu \mathrm{g} / \mathrm{dL}$. Interestingly, at the time of hematoma diagnosis, the latter 6 patients had similar serum cortisol levels but lower serum prolactin levels $(7.1 \pm 3.9$ vs $14.1 \pm$ $7.9 \mu \mathrm{g} / \mathrm{L} ; P<0.01)$ than the 17 patients who eventually had normal function. With glucocorticoid therapy and fluid administration (normal saline), serum $\mathrm{Na}+$ levels gradually improved to normal in all who were hyponatremic at

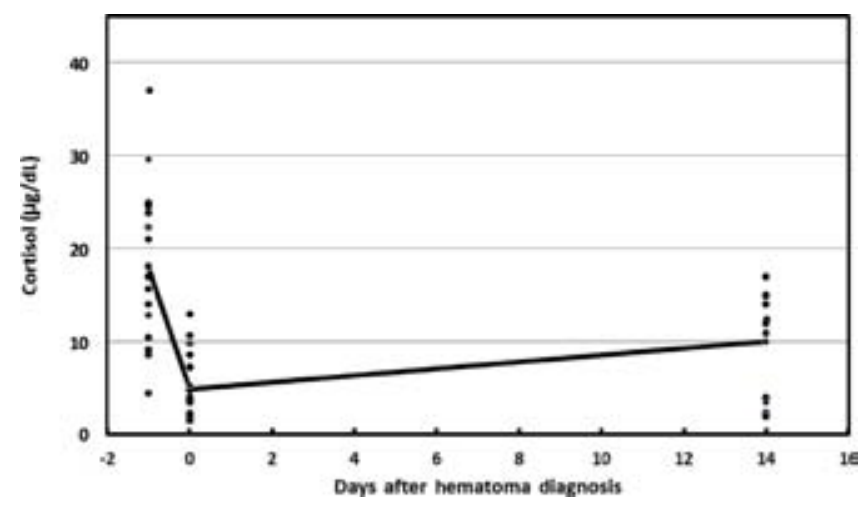

Figure 3

Random serum cortisol levels obtained at the time of hematoma diagnosis (day 0 ), the day before that (day -1 ) and 14-16 days later when all patients were off glucocorticoids for $\geq 2$ days. diagnosis. The 23 patients were followed closely and their pituitary function was re-assessed 3 months after surgery. At that time, the HPA function remained normal in all 17 patients who had documentation of normality after glucocorticoid withdrawal and remained impaired in the remaining 6. Assessment of the rest of pituitary function at 3 months postoperatively revealed impairment in the gonadal (19/23) and thyroidal functions (8/23), while some (14/23) had low plasma IGF1 levels suggestive of decreased $\mathrm{GH}$ secretion. $\mathrm{ADH}$ function as defined by the lack of relevant symptoms, a normal serum, and simultaneously determined urinary $\mathrm{Na}$ and osmolality, was not impaired in any of the 23 patients when tested at 3 months.

Nearly all patients with large tumors (adenomas and RCCs) who did not have clinically significant hematomas ( $n=348$ ) had headaches with variable degrees of intensity during the first 24 postoperative hours, but none had any unexplained visual symptoms, cranial nerve palsies, or alterations in their mental status. The headache in the latter group of patients improved with removal of the nasal packs, usually at $24 \mathrm{~h}$, with minimal discomfort on the second postoperative day. Pituitary function in the latter group of patients was monitored carefully during the first 48 postoperative hours, and there were no demonstrable evidence of changes particularly in HPA function as was previously documented $(3,18)$.

\section{Discussion}

The data presented herein demonstrate that postoperative sellar hematoma is not an infrequent complication of transsphenoidal surgery. It was observed after surgical removal of large adenomas as well as RCC but not in any of the 28 patients who had microadenomas. Postoperative sellar hematoma should not be confused with the well-recognized syndrome of apoplexy involving a pituitary tumor (5) or RCC (12). The latter distinction is supported by evidence from the surgical findings of only a hematoma in patients with the former diagnosis who had surgical decompression and the resolution of the mass effect over time in most patients with glucocorticoid therapy. In reviewing published data, this complication was reported only occasionally $(13,14,15)$ and as case reports at times. The earlier reports on this complication described patients presented with this complication as being similar to that of a mass or pseudo tumor (13). Our data are quite consistent with that description and offer biochemical depiction of the findings.

The data presented herein indicate that the incidence of clinically significant postoperative sellar hematoma 
is approximately 6\% in those with large tumors. It is clearly higher than that reported in the literature. A study involving a survey of nearly 1000 neurosurgeons in the US showed that the complications of transsphenoidal pituitary surgery vary widely and include vascular injuries and hemorrhage into tumor bed in $1-4 \%$ of cases (10). The latter complications are not well defined and may relate to sellar hematoma. As predicted, expert surgeons reported lower complication than those with less experience in that surgery (10). It was interesting to note that anterior pituitary insufficiency was self-reported by even the most experienced surgeons in $7-20 \%$ of patients (10). As this was a self-reporting survey, it would be difficult to ascertain the pathophysiology of hypopituitarism in these patients, and whether this could be a manifestation of unrecognized sellar hematoma. In a recent series where there was an endonasal endoscopic approach to pituitary tumor removal, lower rates of complications including vascular injury and hemorrhage (nearly 1\%) were reported. The latter report did not provide details about the nature or extent of the hemorrhage $(19,20)$.

It is not clear why this complication has not been commonly reported in the past. One possible explanation could be that in many centers, glucocorticoids are administered to all patients, often in large doses in the perioperative period and the latter treatment is tapered gradually over several weeks. Thus, patient would often be receiving higher than physiological doses of glucocorticoids during the critical time when they are at risk of developing this complication. It was interesting to note that nearly onequarter of the patients who developed clinically significant hematomas were receiving ASA or other nonsteroidal agents, although all discontinued such medication over a week before surgery. It may be reasonable to suggest that such medications should be discontinued for more than a week before surgery, especially when the procedure is elective and the mass is large. We did not do any further studies to better characterize the hematological features in these patients. It is also not clear why this complication was not observed in any of the 28 patients who had resection of smaller adenomas (microadenomas).

It would be hard to estimate the exact prevalence of postoperative sellar hematomas solely based on imaging studies as these are not routinely performed in the immediate postoperative period and are usually performed for specific clinical indications such as increasing headaches, alterations in mental status, and visual changes. Imaging studies done shortly after transsphenoidal pituitary surgery often reveal a variety of changes at the surgical site, including evidence of blood or blood products and some edema; features often referred to as postoperative changes. Thus, it is highly likely that following surgical removal of a pituitary mass, blood oozing might be evident at the surgical site in many patients, but this would not be clinically apparent in the majority. It is therefore important to point out that our estimate for the development of postoperative sellar hematoma refers only to those that are symptomatic, clinically significant, and will thus justify confirmation with an imaging study.

The biochemical data obtained before and soon after the onset of symptoms are quite important as it sheds some light not only on the prevalence of this complication but also on the potential mechanism of hypopituitarism in this setting. We focused on the assessment of HPA function as this is highly activated in the perioperative period (3), and is clinically important at that time. Furthermore, the short half-life of the components of the HPA function (particularly ACTH and cortisol) in the circulation makes assessment of their function easier to recognize in the acute setting. In addition, we assessed prolactin levels in these patients because its secretion is regulated distinctly different from other pituitary hormones including ACTH. The latter physiological feature helps in considering potential mechanisms for hypopituitarism in this setting just as it was in proposing the pathophysiology of loss of pituitary function in patients with large sellar tumors $(2,3,21,22)$.

We noted that some of the clinical symptoms observed in patients with postoperative sellar hematoma were due to a mass effect such as the headaches and visual manifestations, wheras others could be caused by loss of pituitary function and particular HPA function. It is important to point out that the onset of hypopituitarism in this setting is rather acute as demonstrated by the rapid decline in plasma ACTH and all ACTH-dependent adrenal steroids: cortisol, DHEA, and DHEA-S. These changes were associated with a rise, albeit mild, in serum prolactin level. Such alterations are consistent with the compression of portal vessels and/or the pituitary stalk, likely by the expanding hematoma. This mechanism is reminiscent of the proposed chronic effects of perisellar mass lesions such as tumors on pituitary function $(2,3,21,22,23)$. The rapid recovery in most patients after discontinuation of glucocorticoid therapy is consistent with the postulated mechanism and indicates that pituitary cells remained viable after the development of hematoma in most patients.

The improvement in clinical symptoms was quite rapid and was observed within $24 \mathrm{~h}$ of the administration of glucocorticoids. Since the improvement in 
headaches and visual symptoms was quickly noted, it is likely that the glucocorticoid administration helped to minimize the edema and swelling in the perisellar region. The recovery of HPA function after the withdrawal of glucocorticoid therapy indicated that the loss of pituitary function in this setting was not only acute in onset but was also reversible in the majority of patients. The persistence of ACTH deficiency in the minority of patients suggested that ischemic changes and/or necrosis of some of the normal pituitary tissue may have accompanied the development of hematomas. We did not attempt to measure intrasellar pressure in these patients as only four subjects had repeat surgery. Despite the lack of definitive data, it is reasonable to speculate that a rise in intrasellar pressure mediated some of the manifestations of postoperative hematoma just as it was demonstrated to have a pathophysiological role in the development of headaches and hypopituitarism $(24,25)$.

In summary, our data demonstrate that approximately $6 \%$ of patients who had transsphenoidal surgical resection of large $(>1 \mathrm{~cm})$ pituitary adenomas or RCC developed clinically significant postoperative hematoma at the surgical site. The development of postoperative sellar hematoma resulted in a mass effect that manifested clinically as headaches, visual and neurological symptoms as well as acute onset of hypopituitarism. The latter was characterized by a drastic reduction in the secretion of ACTH and ACTH-dependent steroids (cortisol, DHEA, and DHEA-S) and was associated with a rise in serum prolactin levels. The rapid onset and the reversibility of hypopituitarism suggest that compression of portal vessels/pituitary stalk by the hematoma was the primary mechanism for pituitary dysfunction. Prompt recognition and glucocorticoid administration led to the resolution of clinical manifestations and recovery of pituitary function in most patients.

\section{Declaration of interest}

The authors declare that there is no conflict of interest that could be perceived as prejudicing the impartiality of the research reported.

\section{Funding}

This work was supported by a local departmental grant to the Division of Endocrinology

\section{References}

1 Hout WM, Arafah BM, Salazar R \& Selman WR. Evaluation of the hypothalamic- pituitary- adrenal axis immediately after pituitary adenomectomy: is perioperative steroid therapy necessary? Journal of Clinical Endocrinology \& Metabolism 198866 1208-1212.

2 Arafah BM. Reversible hypopituitarism in patients with large nonfunctioning pituitary adenomas. Journal of Clinical Endocrinology \& Metabolism 198662 1173-1179. (doi:10.1210/jcem-62-6-1173)

3 Arafah BM, Kailani SH, Nekl KE, Gold RS \& Selman WR. Immediate recovery of pituitary function after transsphenoidal resection of pituitary macroadenomas. Journal of Clinical Endocrinology \& Metabolism 199479 348-354.

4 Arafah BM, Harrington JF, Madhun ZT \& Selman WR. Improvement of pituitary function after surgical decompression for pituitary tumor apoplexy. Journal of Clinical Endocrinology \& Metabolism 199071 323-328.(doi:10.1210/jcem-71-2-323)

5 Nawar R, Abdel-Mannan D, Selman WR \& Arafah BM. Pituitary tumor apoplexy: a review. Journal of Intensive Care Medicine 200823 75-90. (doi:10.1177/0885066607312992)

6 Aho CJ, Liu C, Zelman V, Couldwell WT \& Weiss MH. Surgical outcomes in 118 patients with Rathke cleft cysts. Journal of Neurosurgery 2005102 189-193. (doi:10.3171/jns.2005.102.2.0189)

7 Binning MJ, Liu JK, Gannon J, Osborn AG \& Couldwell WT. Hemorrhagic and nonhemorrhagic Rathke cleft cysts mimicking pituitary apoplexy. Journal of Neurosurgery 2008108 3-8. (doi:10.3171/JNS/2008/108/01/0003)

8 Couldwell WT \& Weiss MH. Surgical management of Rathke's cleft cysts. Current Opinion in Endocrinology Diabetes and Obesity 200613 351-355.

9 Chaiban J, Abdelmannan D, Cohen M, Selman WR \& Arafah BM. Rathke's cyst apoplexy: a newly characterized distinct clinical entity. Journal of Neurosurgery 2011114 318-324. (doi:10.3171/2010.5.JNS091905)

10 Ciric I, Ragin A, Baumgartner C \& Pierce D. Complications of transsphenoidal surgery: Results of national survey, review of the literature and personal experience. Neurosurgery 199740 225-237. (doi:10.1097/00006123-199702000-00001)

11 Turner HE, Adams CB \& Wass JA. Pituitary tumours in the elderly: a 20 year experience. European Journal of Endocrinology 1999140 383-389. (doi:10.1530/eje.0.1400383)

12 Barzaghi LR, Losa M, Giovanelli M \& Mortini P. Complications of transsphenoidal surgery in patients with pituitary adenoma: experience at a single centre. Acta Neurochirurgica: The European Journal of Neurosurgery 2007149 877-886. (doi:10.1007/s00701007-1244-8)

13 Veldhuis JD \& Santen RJ. Pituitary pseudotumor: Mimicry of recurrent prolactinomas by a chronic intrasellar hematoma. Archives of Internal Medicine 1979139 1309-1311. (doi:10.1001/archinte.1979.03630480081025)

14 Hojo M, Goto M \& Miyamoto S. Chronic expanding pituitary hematoma without rebleeding after pituitary apoplexy. Surgical Neurology International $2013 \mathbf{4}$ 41-44.

15 Atkinson JLD, Nippoldt TB \& Koellert K. Reoperation for sella hematoma after pituitary surgery. Clinical Endocrinology 200868 413-415.

16 Griffith HB \& Veerapen R. A direct transnasal approach to the sphenoid sinus. Technical note. Journal of Neurosurgery 198766 140-142. (doi:10.3171/jns.1987.66.1.0140)

17 Al-Aridi R, Abdelmannan D \& Arafah BM. Biochemical diagnosis of adrenal insufficiency: the added value of dehydroepiandrosterone sulfate (DHEA-S) measurements. Endocrine Practice 201117 261-270. (doi:10.4158/EP10262.RA)

18 Sayyed Kassem L \& El Sibai K, Chaiban J Abdelmannan D, Arafah BM. Measurements of serum DHEA and DHEA-sulphate levels improve the accuracy of the low dose cosyntropin test in the diagnosis of central adrenal insufficiency. Journal of Clinical Endocrinology \& Metabolism $2012973655-3663$.

19 Dehdashti A, Ganna A, Karabatsou K \& Gentilli F. Pure endoscopic endonasal approach for pituitary adenomas: early surgical results 
in 200 patients and comparison with previous microsurgical series. Neurosurgery 200862 1006-1017.

20 Wagenmakers MAEM, Boogarts HD, Roerink SHPP, Timmers HJLM, Stikkelbroeck NMML, Smit JWA, van Lindert EJ, Natea-Maier RT, Grotenhuis JA \& Hermus ARMM. Endoscopic transsphenoidal pituitary surgery: a good and safe treatment option for Cushing's disease even in case of macroadenomas or invasive adenomas. European Journal of Endocrinology 2013169 329-337. (doi:10.1530/EJE-13-0325)

21 Anonymous. Reversible hypopituitarism (Editorial). The Lancet 1991 337276.

22 Arafah BM \& Nasrallah MP. Pituitary tumors: pathophysiology, clinical manifestations and management. Endocrine-Related Cancer 20018 287-305. (doi:10.1677/erc.0.0080287)
23 Bland ES, Lees PD \& Pickard JD. Endocrine function in the stalk compression syndrome. Neuroendocrinology 199052 147-151.

24 Arafah BM, Prunty D, Ybarra J, Hlavin ML \& Selman WR. The dominant role of increased intrasellar pressure in the pathogenesis of hypopituitarism, hyperprolactinemia and headaches in patients with pituitary adenomas. Journal of Clinical Endocrinology \& Metabolism 200085 1789-1793.

25 Zayour DH, Selman WR \& Arafah BM. Extreme elevation in intrasellar pressure in patients with pituitary tumor apoplexy: relation to pituitary function. Journal of Clinical Endocrinology \& Metabolism 2004 89 5649-5654.

Received 29 September 2015

Revised version received 9 December 2015

Accepted 4 February 2016 